\title{
Conformal Quantum Field Theory and Half-Sided Modular Inclusions of von-Neumann-Algebras
}

\section{Hans-Werner Wiesbrock ${ }^{\star}$}

Institut für Theoretische Physik, FU Berlin, D-14195, Berlin, Germany

Received: 5 January, 1993/in revised form: 22 March, 1993

Abstract: Let $\mathscr{N}, \mathscr{M}$ be von-Neumann-Algebras on a Hilbert space $\mathscr{H}, \Omega$ a common cyclic and separating vector. Assume $\Omega$ to be cyclic and separating also for $\mathscr{N} \cap \mathscr{M}$. Denote by $J_{\mathscr{M}}, J_{\mathcal{N}}$ the modular conjugations to $(\mathscr{M}, \Omega), \Delta_{\mathscr{M}}$ and $\Delta_{\mathcal{N}}$ the associated modular operators. If

$$
\begin{array}{ll}
\Delta_{\mathscr{M}}^{-i t}(\mathscr{N} \cap \mathscr{M}) \Delta_{\mathscr{M}}^{i t} \subset(\mathscr{N} \cap \mathscr{M}) & \text { for all } t \geqq 0, \\
\Delta_{\mathscr{N}}^{i t}(\mathscr{N} \cap \mathscr{M}) \Delta_{\mathcal{N}}^{-i t} \subset(\mathscr{N} \cap \mathscr{M}) & \text { for all } t \geqq 0,
\end{array}
$$

and

$$
J_{\mathscr{M}} \mathcal{N} J_{\mathscr{M}}=\mathscr{N},
$$

these data define in a canonical way a conformal quantum field theory on a circle. Conversely, the chiral part of a conformal quantum field theory in two dimensions always yields such data in a natural way.

\section{Introduction}

It is well known that conformal quantum field theory in two dimensions factor into two chiral conformal theories on the lightrays, see [5]. In the framework of Algebraic Quantum Field Theory, see [6], they are described by a net $\mathscr{A}(I)$ of von-Neumann algebras, indexed by the set $\mathscr{J}$ of proper intervals $I \subset S^{1}$, with

1. $\mathscr{A}(I) \subset \mathscr{A}(J)$ if $I \subset J$ (isotony)

2. $\mathscr{A}(I) \subset \mathscr{A}(J)^{\prime}$ if $I \cap J=\emptyset$ (locality),

acting on a Hilbert space $\mathscr{H}$. On $\mathscr{H}$ there is given a strongly continuous unitary positive energy representation $U$ of $S l(2, \mathbf{R}) / \mathbf{Z}_{2}$ with a unique invariant vacuum vector $\Omega$. The net transforms covariantly under this representation.

^ EMAIL: WIESBROCK @ risc4.physik.fu-berlin.de

Partly supported by the DFG, SFB 288 Differentialgeometrie und Quantenphysik 
Let $\mathscr{M}=\mathscr{M}(\cap)$ be the algebra of the upper half circle and $\mathscr{N}=\mathscr{M}(\subset)$ be of the left half circle. Assuming the net to be generated by Wightman Fields, which generically seems to be case, [7], we may apply the results of Bisognano and Wichman, [1], to $\mathscr{M}$ and $\Omega$. They showed that the modular group $\sigma_{\mathscr{M}}^{t}$ associated with $(\mathscr{M}, \Omega)$ acts geometrically as a Lorentz boost. Especially one gets

$$
\text { a) } \sigma_{\mathscr{M}}^{-t}(\mathscr{N} \cap \mathscr{M}) \subset \mathscr{N} \cap \mathscr{M} \text { for all } t \geqq 0 \text {, }
$$

moreover by the Reeh-Schlieder property

b) $\Omega$ is a standard vector for $\mathscr{N} \cap \mathscr{M}$.

By the $\operatorname{Sl}(2, R) / Z_{2}$-covariance this is also true for $\mathscr{M}$ exchanged by $\mathscr{N}$ and $t \leqq 0$. The work of Borchers, [2], shows that $J_{\mathscr{N}}$ acts on the net like a reflection. Especially one gets

$$
\text { c) } J_{\mathscr{N}} \mathscr{M} J_{\mathcal{N}}=\mathscr{M} \text {. }
$$

In this paper we conversely show that any pair of von-Neumann-algebras $\mathcal{N}$ and $\mathscr{M}$ with a common cyclic and separating vector $\Omega$ obeying the above relations in a canonical way gives rise to a conformal field theory on the circle in the sense described above. The crucial observation is that half-sided modular inclusions carry a rich symmetry. For the reader's convenience we recall some results obtained in $[9,10]$.

\section{Half-Sided Modular Inclusions and Symmetries}

Assume $\tilde{\mathscr{M}} \subset \mathscr{M}$ to be von-Neumann-Algebras acting on a Hilbert space $\mathscr{H}$, and $\Omega \in \mathscr{H}$ a common cyclic and separating vector. Let $\Delta_{\mathscr{M}}^{-i t} \tilde{\mathscr{M}} \Delta_{\tilde{M}}^{i t} \subset \tilde{\mathscr{M}}$ for all $t \geqq 0$. We call such an inclusion $(\tilde{\mathscr{M}} \subset \mathscr{M}, \Omega)$-half-sided modular, see $[9,10]$. If one changes $t \geqq 0$ to $t \leqq 0$, we call it + half-sided modular, abbreviated by $\mp$-hsm. Denote by $\Delta_{\mathscr{M}}, \Delta_{\tilde{\mathscr{M}}}$ the modular operators associated with $(\mathscr{M}, \Omega)$ and $(\tilde{\mathscr{M}}, \Omega)$, respectively. For such a situation we proved in [9] the following

Theorem 1. Let $(\tilde{\mathscr{M}} \subset \mathscr{M}, \Omega)$ be a F-half-sided modular inclusion, $\Delta_{\mathscr{M}}, \Delta_{\tilde{M}}$ the modular operators associated with $(\mathscr{M}, \Omega)$ and $(\tilde{\mathscr{M}}, \Omega)$, respectively. Assume

$$
\Delta_{\mathscr{M}}^{i t} \tilde{\mathscr{M}} \Delta_{\mathscr{M}}^{-i t} \subset \tilde{\mathscr{M}} \text { for all } \mp t \geqq 0 \text {. }
$$

Then
a) $\frac{1}{2 \pi}\left(\ln \left(\Delta_{\tilde{M}}\right)-\ln \left(\Delta_{\mathscr{M}}\right)\right) \geqq 0$

is essentially selfadjoint. Denote $U(a), a \in R$, the unitary group on $\mathscr{H}$ with generator $\frac{1}{2 \pi}\left(\ln \left(\Delta_{\tilde{M}}\right)-\ln \left(\Delta_{\mathscr{M}}\right)\right)^{-}$. Then

b) $\Delta_{\mathscr{M}}^{i t} U(a) \Delta_{\mathscr{M}}^{-i t}=\Delta_{\mathscr{M}}^{i t} U(a) \Delta_{\tilde{\tilde{M}}}^{i t}=U\left(e^{\mp 2 \pi t} a\right)$ for all $t, a \in R$,

c) $J_{\mathscr{M}} U(a) J_{\mathscr{M}}=J_{\tilde{M}} U(a) J_{\tilde{M}}=U(-a)$ for all $a \in R$,

d) $\Delta_{\tilde{M}}^{i t} \mathscr{M} \Delta_{\tilde{\tilde{M}}}^{i t} \subset \mathscr{M}$ for all $\mp t \geqq 0$,

e) $\tilde{\mathscr{M}}=U( \pm 1) \mathscr{M} U(\mp 1)$. 
Proof. See Theorem 3, Cor. 6 and Cor. 7 of [9].

This theorem shows already that in the case of half-sided modular inclusions of von-Neumann-algebras their modular operators yield a representation of the two dimensional subgroup of $S l(2, R) / Z_{2}$ generated by the translations and dilatations.

Theorem 2. Let $\mathscr{M}, \mathscr{M}_{1}, \mathscr{M}_{2}$ be von-Neumann-algebras on a Hilbert space $\mathscr{H}, \Omega \in \mathscr{H}$ a common cyclic and separating vector. Denote by $\Delta_{\mathscr{M}}, \Delta_{\mathscr{M}_{1}}, \Delta_{\mathscr{M}_{2}}$ the modular operators to $(\mathscr{M}, \Omega)\left(\mathscr{M}_{1}, \Omega\right),\left(\mathscr{M}_{2}, \Omega\right)$. Assume

1. $\left(\mathscr{M}_{1} \subset \mathscr{M}, \Omega\right)-$ hsm,

2. $\left(\mathscr{M}_{2} \subset \mathscr{M}, \Omega\right)+$ hsm,

3. $\left(\mathscr{M}_{2} \subset \mathscr{M}_{1}^{\prime}, \Omega\right)$ - hsm,

where the prime indicates the commutant. Then

$$
\Delta_{\mathscr{M}}^{i t}, \Delta_{\mathscr{M}_{1}}^{i r}, \Delta_{\mathscr{M}_{2}}^{i s}, t, r, s \in \mathbb{R}
$$

generate a representation of the universal covering group $\operatorname{Sl}(\tilde{2}, R)$. Denote by $\mathscr{V}$ this representation. For the image of the rotation by $\pi$ in the first sheet of $\operatorname{Sl}(\tilde{2}, R)$, denoted by $\operatorname{rot}(\pi, 1) \in \operatorname{Sl}(\tilde{2}, R)$, one computes

$$
\mathscr{V}(\operatorname{rot}(\pi, 1))=J_{\mathscr{M}}\left(\Delta_{\mathscr{M}_{1}}^{\frac{-\ln 2}{2 \pi}} J_{\mathscr{M}_{2}} \Delta_{\mathscr{M}_{1}}^{\frac{\ln 2}{2 \pi}}\right)
$$

Proof. See [10, Lemma 3 and Lemma 4 ff].

In the next section we will apply these results in order to formulate a vonNeumann algebraic characterization of conformal quantum field theories on a circle.

\section{Half-Sided Modular Inclusions and Conformal Field Theory}

Let $\mathscr{N}, \mathscr{M}, \mathscr{N} \cap \mathscr{M}$ be von-Neımann algebras on a Hilbert space, $\Omega$ a common cyclic and separating vector. Assume

1. $((\mathscr{N} \cap \mathscr{M}) \subset \mathscr{M}, \Omega)$ is - half-sided-modular,

2. $((\mathscr{N} \cap \mathscr{M}) \subset \mathscr{N}, \Omega)$ is + half-sided-modular,

3. $J_{\mathscr{N}} \mathscr{M} J_{\mathscr{N}}=\mathscr{M}$,

where the modular objects are indexed by the related von-Neumann algebra as before. ${ }^{1}$ Such a situation naturally occurs in Bisognano-Wichmann nets of conformal quantum field theories on a circle, as was mentioned in the introduction. $\mathscr{M}$ denotes in this case the observable algebra of the upper half circle, $\mathscr{N}$ of the left

\footnotetext{
1 The importance of the third relation was already noticed by B. Schroer in [8], where the reader can find some preliminary ideas on the representation of conformal field theories from pairs of von-Neumann algebras
} 
half circle. We will show that conversely such a pair of von-Neumann algebras yield in a canonical way a conformal quantum field theory on the circle. ${ }^{2}$

Theorem 3. Let $(\mathscr{N}, \mathscr{M}, \Omega)$ be as above. Then these data define in a canonical way a local net of von-Neumann algebras on $S^{1}$, which transforms co-variantly under $\operatorname{Sl}(2, R) / Z_{2}$. The representation is of positive energy, i.e. we get a conformal quantum field model on $S^{1}$. This net fulfills Haag Duality and the Reeh-Schlieder property.

Proof. We will prove this theorem in several steps. Firstly we will show that

$$
\Delta_{\mathscr{M}}^{i t}, \Delta_{\mathscr{N} \cap \mathscr{M}}^{i r}, \Delta_{\mathcal{N}^{\prime} \cap \mathscr{M}}^{i s}, t, r, s \in \mathbb{R}
$$

generate a representation of the group $\operatorname{Sl}(2, R) / Z_{2}$. This will be done by applying Theorem 2 to $\mathscr{M}_{1}=\mathscr{N} \cap \mathscr{M}, \mathscr{M}_{2}=\mathscr{N}^{\prime} \cap \mathscr{M}$. Secondly we will define a $S l(2, R) / Z$ covariant net on $S^{1}$ by using the modular representation of the Moebius group together with defining $\mathscr{M}$ to be the algebra associated to the upper half circle. In the last step we will show that this net is isotonic and local.

In order to apply Theorem 2 we have to prove

a) $((\mathscr{N} \cap \mathscr{M}) \subset \mathscr{M}, \Omega)$ is - hsm,

b) $\left(\left(\mathscr{N}^{\prime} \cap \mathscr{M}\right) \subset \mathscr{M}, \Omega\right)$ is $+\mathrm{hsm}$,

c) $\left(\left(\mathscr{N}^{\prime} \cap \mathscr{M}\right) \subset\left(\mathscr{N}^{\prime} \vee \mathscr{M}^{\prime}\right), \Omega\right)$ is $-\mathrm{hsm}$.

a) is just one of the assumptions, (1).

b) Notice that by assumption 3 we get $\left[J_{\mathscr{N}}, \Delta_{\mathscr{M}}\right]=0$. Applying $\operatorname{Ad}\left(J_{\mathscr{N}}\right)$ to the - hsm inclusion a), one immediately obtains b).

c) is the most difficult one. Applying Theorem 1 to the + hsm inclusion $((\mathscr{N} \cap \mathscr{M}) \subset \mathscr{N}, \Omega)$, one gets a one parameter group

$$
\tilde{U}(a):=\exp \left(\frac{1}{2 \pi} a\left(\ln \Delta_{\mathscr{N} \cap \mathscr{M}}-\ln \Delta_{\mathcal{N}}\right)\right)
$$

with

$$
\mathscr{N} \cap \mathscr{M}=\tilde{U}(-1) \mathscr{N} \tilde{U}(1)
$$

and

$$
\Delta_{\left(\mathscr{N}^{\prime} \vee \mathscr{M}^{\prime}\right)}^{i t}=\tilde{U}(-1) \Delta_{\mathcal{N}}^{-i t} \tilde{U}(1)=\tilde{U}\left(-1+e^{-2 \pi t}\right) \Delta_{\mathscr{N}}^{-i t} .
$$

Therefore we get

$$
\begin{aligned}
\operatorname{Ad}\left(\Delta_{\mathscr{N}^{\prime} \vee \mathscr{M}}^{i t}\right)\left(\mathscr{N}^{\prime} \cap \mathscr{M}\right) & =\operatorname{Ad}\left(\tilde{U}\left(-1+e^{-2 \pi t}\right) \Delta_{\mathcal{N}}^{-i t}\right)\left(\mathscr{N}^{\prime} \cap \mathscr{M}\right) \\
& =\operatorname{Ad}\left(\tilde{U}\left(-1+e^{-2 \pi t}\right) \Delta_{\mathcal{N}^{t}}^{-i t} J_{\mathscr{N}}\right)(\mathscr{N} \cap \mathscr{M})
\end{aligned}
$$

by assumption 3 , and using Theorem 1c),

$$
=\operatorname{Ad}\left(J_{\mathscr{N}} \tilde{U}\left(1-e^{-2 \pi t}\right) \Delta_{\mathscr{N}}^{-i t}\right)(\mathscr{N} \cap \mathscr{M}) .
$$

${ }^{2}$ In an earlier version of this paper the author proposed different conditions on the relative position of two algebras $\mathcal{N}, \mathscr{M}$, in order to characterize uniquely a conformal net on the circle. D. Buchholz pointed out to us a serious error in the previous construction of the net. The author thanks D. Buchholz warmly for his valuable comments 
By assumption $2((\mathscr{N} \cap \mathscr{M}) \subset \mathscr{N}, \Omega)$ is + hsm, and Theorem 1b), e) shows

$$
\operatorname{Ad}(\tilde{U}(a))(\mathscr{N} \cap \mathscr{M}) \subset(\mathscr{N} \cap \mathscr{M}) \text { for } a \leqq 0 .
$$

Putting everything together we get

$$
\operatorname{Ad}\left(\Delta_{\left(\mathscr{N}^{\prime} \vee \mathscr{M}^{\prime}\right)}^{i t}\right)\left(\mathscr{N}^{\prime} \cap \mathscr{M}\right) \subset \operatorname{Ad}\left(J_{\mathscr{N}}\right)(\mathscr{N} \cap \mathscr{M})=\mathscr{N}^{\prime} \cap \mathscr{M}
$$

for $t \leqq 0$. Therefore we can apply Theorem 2 .

In order to reduce the symmetry to the Moebius group $S l(2, R) / Z_{2}$, we make use of (4) and calculate

$$
\begin{aligned}
& \Delta_{\mathscr{N}}^{\frac{-i \ln 2}{2 \pi}} \mathscr{M}\left(\mathscr{N}^{\prime} \cap \mathscr{M}\right) \Delta_{\mathscr{N} \cap}^{\frac{\operatorname{in} 2}{2 \pi} \cap \mathscr{M}}=\operatorname{Ad}\left(\Delta_{\mathscr{N}}^{\frac{-i \ln 2}{2 \pi}} \tilde{U}(-1)\right)\left(\mathscr{N}^{\prime} \cap \mathscr{M}\right) \\
& =\operatorname{Ad}\left(\Delta_{\mathcal{N}}^{\frac{-i \ln 2}{2 \pi}} \tilde{U}(-1) J_{\mathscr{N}}\right)(\mathscr{N} \cap \mathscr{M}) \\
& =\operatorname{Ad}\left(\Delta_{\mathscr{N}}^{\frac{-i \ln 2}{2 \pi}} J_{\mathscr{N}} \tilde{U}(1)\right)(\mathscr{N} \cap \mathscr{M}) .
\end{aligned}
$$

By Theorem 1e) we get

$$
=\operatorname{Ad}\left(\Delta_{\mathscr{N}}^{\frac{-i \ln 2}{2 n}} J_{\mathscr{N}}\right)(\mathscr{N})=\mathscr{N}^{\prime}
$$

Therefore

$$
\Delta_{\mathscr{N} \cap}^{\frac{-i \ln 2}{2 \pi}} J_{\left(\mathscr{N}^{\prime} \cap \mathscr{M}\right)} \Delta_{\mathscr{N} \cap \mathscr{M} n}^{\frac{i n 2}{2 \pi}}=J_{\mathscr{N}} .
$$

By assumption 3 we have $\left[J_{\mathscr{N}}, J_{\mathscr{M}}\right]=0$, i.e. by Theorem 2 the rotation by $2 \pi$ lies in the kernel of the representation of $\operatorname{Sl}(\tilde{2}, R)$. The symmetry reduces to the Moebius group $\operatorname{Sl}(2, R) / Z_{2}$.

Let us denote the representation of the Moebius group $\operatorname{Sl}(2, R) / Z_{2}$ by $\mathscr{V}$. The above calculation especially shows

$$
\mathscr{V}(\operatorname{rotation}(\pi))=J_{\mathcal{N}} J_{\mathscr{M}},
$$

where rotation is the 1-parameter subgroup of rigid rotations in $\operatorname{Sl}(2, R) / Z_{2}$. Next we want to define a net of algebras indexed by the proper intervals of $S^{1}$.

Let $(a, b) \subset S^{1}$ be a proper interval. There exists an element $g_{(a, b)} \in S l(2, R) / Z_{2}$ which maps the upper half circle $(1,-1)$ onto $(a, b)$,

$$
g_{(a, b)}((1,-1))=(a, b) \text {. }
$$

Let us define

$$
\mathscr{M}(a, b):=\mathscr{V}\left(g_{(a, b)}\right) \mathscr{M} \mathscr{V}\left(g_{(a, b)}\right)^{*} .
$$

This definition does not depend on the special choice of $g_{(a, b)} \in S l(2, R) / Z_{2}$ with the above property. To see this notice that elements of the Möbius group which map the upper half circle onto itself are dilatations. Starting from $g( \pm 1)= \pm 1$ this is a one line calculation. Then it is easily seen that two elements $g_{1}, g_{2} \in \operatorname{Sl}(2, R) / Z_{2}$ mapping the upper half circle onto $(a, b)$ can only differ in the dilatation factor. But the dilatations are represented by the modular group of $\mathscr{M}$,

$$
\mathscr{V}(\text { dilatation }(\lambda))=\Delta_{\mathscr{M}}^{\frac{-\sin \lambda}{2 \pi}},
$$

which proves the well definedness of $\mathscr{M}(a, b)$. By the very construction the net $\mathscr{M}(a, b)$ transforms covariantly w.r.t. to the representation $\mathscr{V}$ of $S l(2, R) / Z_{2}$.

Let us make a simple but crucial observation. By the above calculation we know $\mathscr{V}(\operatorname{rotation}(\pi))=J_{\mathscr{N}} J_{\mathscr{M}}$. Moreover it is easily seen that

$$
g_{(b, a)}:=g_{(a, b)} \cdot \operatorname{rotation}(\pi)
$$


maps the upper half circle onto the complement $S^{1} \backslash[a, b]$. This yields

$$
\begin{aligned}
\mathscr{M}(b, a) & =\mathscr{V}\left(g_{(b, a)}\right) \mathscr{M} \mathscr{V}\left(g_{(b, a)}\right)^{*} \\
& =\mathscr{V}\left(g_{(a, b)}\right) \mathscr{V}(\operatorname{rotation}(\pi)) \mathscr{M} \mathscr{V}(\operatorname{rotation}(\pi))^{*} \mathscr{V}\left(g_{(a, b)}\right)^{*} \\
& =\mathscr{V}\left(g_{(a, b)}\right) J_{\mathscr{M}} J_{\mathscr{N}} \mathscr{M} J_{\mathscr{N}} J_{\mathscr{M}} \mathscr{V}\left(g_{(a, b)}\right)^{*} \\
& =\mathscr{V}\left(g_{(a, b)}\right) \mathscr{M}^{\prime} \mathscr{V}\left(g_{(a, b)}\right)^{*} \\
& =\left(\mathscr{V}\left(g_{(a, b)}\right) \mathscr{M} \mathscr{V}\left(g_{(a, b)}\right)^{*}\right)^{\prime}=(\mathscr{M}(a, b))^{\prime}
\end{aligned}
$$

Let us prove isotony of the net. For this one notices that the translations are represented by

$$
\mathscr{V}(\operatorname{translation}(a)):=\exp \left(\frac{i a}{2 \pi}\left(\ln \Delta_{\mathcal{N} \cap \mathscr{M}}-\ln \Delta_{\mathscr{M}}\right)\right),
$$

and Theorem 1 implies

$$
\operatorname{Ad}(\mathscr{V}(\operatorname{translation}(a)))(\mathscr{M}) \subset \mathscr{M} \quad \text { for } a \geqq 0 .
$$

Therefore

$$
\mathscr{M}(a,-1) \subset \mathscr{M}(b,-1) \text { for }(a,-1) \subset(b,-1),
$$

and using the rotations this proves

$$
\mathscr{M}(a, c) \subset \mathscr{M}(b, c) \text { for }(a, c) \subset(b, c) .
$$

Passing to commutants and making use of relation (17) completes the proof of isotony,

$$
\mathscr{M}(c, a) \subset \mathscr{M}(c, b) \text { for }(c, a) \subset(c, b) .
$$

What is left to be proven is locality. But now this is nearly trivial. Let $(a, b),(c, d) \subset S^{1}$ be proper intervals with empty intersection. Then $(a, b) \subset(d, c)$, and by isotony and relation (17),

$$
\mathscr{M}(a, b) \subset \mathscr{M}(d, c)=(\mathscr{M}(c, d))^{\prime} .
$$

The rest of the theorem follows easily.

Let me finish with two remarks.

Remark 1. Consider $(\mathscr{N}, \mathscr{M}, \Omega)$ as in Theorem 3. Then in particular $((\mathscr{N} \cap \mathscr{M})$ $\subset \mathscr{M}, \Omega)$ is a half-sided modular standard inclusion, i.e. $\Omega$ is also cyclic and separating for $(\mathscr{N} \cap \mathscr{M})^{\prime} \cap \mathscr{M}$. We can apply Theorem 2 to $\mathscr{M}_{1}=\mathscr{N} \cap \mathscr{M}$, $\mathscr{M}_{2}=(\mathscr{N} \cap \mathscr{M})^{\prime} \cap \mathscr{M}, \mathscr{M}=\mathscr{M}$, as it was shown in [10]. Again we get a representation of $S l(2, R) / Z_{2}$ by the modular groups $\Delta_{(\mathscr{N} \cap \mathscr{M})}, \Delta_{(\mathscr{N} \cap \mathscr{M})^{\prime} \cap \mathscr{M}}, \Delta_{\mathscr{M}}$, see $[10]$. Define

$$
U:=J_{\mathcal{N}^{\prime} \cap \mathscr{M}} J_{(\mathscr{N} \cap \mathscr{M})^{\prime} \cap \mathscr{M}} .
$$

Using the various commutation relations one easily sees that

$$
[\mathscr{V}(\operatorname{translation}(a)), U]=0 \text { for all } a \in R \text {. }
$$

Furthermore one gets

$$
U=1 \Leftrightarrow\left(\mathscr{N}^{\prime} \cap \mathscr{M}\right)=\left(\mathscr{N}^{\prime} \vee \mathscr{M}^{\prime}\right) \cap \mathscr{M},
$$

i.e. strong additivity of the net, see [3]. Let me prove the second part. 
Denote

$$
\begin{aligned}
& U(a)=\exp \left(i a\left(\ln \Delta_{\mathscr{N}^{\prime} \cap \mathscr{M}^{\prime}}-\ln \Delta_{\mathscr{M}}\right)\right), \\
& \tilde{U}(a)=\exp \left(i a\left(\ln \Delta_{\left(\mathscr{N}^{\prime} \vee \mathscr{M}^{\prime}\right) \cap \mathscr{M}}-\ln \Delta_{\mathscr{M}}\right)\right) .
\end{aligned}
$$

Then we get from Theorem 1 and the assumptions,

$$
\begin{aligned}
\Delta_{(\mathscr{N} \cap \mathscr{M})^{\prime} \cap \mathscr{M}}^{i t} & =\tilde{U}(-1) \Delta_{\mathscr{M}}^{i t} \tilde{U}(1) \\
& =\Delta_{\mathscr{M}}^{i \frac{\ln 2}{2 \pi}} \tilde{U}(-2) \Delta_{\mathscr{M}}^{i t} \tilde{U}(2) \Delta^{-i \frac{\ln 2}{2 \pi}} .
\end{aligned}
$$

But from $U=1$ we conclude

$$
\begin{aligned}
\tilde{U}(2) & =J_{(\mathscr{N} \cap \mathscr{M})^{\prime} \cap \mathscr{M}_{\mathscr{M}}} J_{\mathscr{N}^{\prime} \cap \mathscr{M}} J_{\mathscr{M}} \\
& =U(2)
\end{aligned}
$$

and therefore

$$
\Delta_{(\mathscr{N} \cap \mathscr{M})^{\prime} \cap \mathscr{M}}^{i t}=\Delta_{\mathscr{N}^{\prime} \cap \mathscr{M}}^{i t} .
$$

Now $\left(\mathscr{N}^{\prime} \cap \mathscr{M}\right) \subset(\mathscr{N} \cap \mathscr{M})^{\prime} \cap \mathscr{M}$, and the above equality proves equality of the algebras.

The converse is trivial.

Remark 2. We did not use any factor property in this work. In the case $\mathscr{M}$ is a factor we proved in [9] that $\mathscr{M}$ has to be of type $I I I_{1}$. It was also shown in [9] that in this case the associated conformal field theory has a unique translation invariant vector, i.e. a unique vacuum vector.

Acknowledgement. I would like to thank C.-P. Staszkiewicz for the many fruitful discussions, H.-J. Borchers for critical remarks and his beautiful work [2], which initiated this area of discussion, and B. Schroer for his stimulating interest and discussions.

\section{References}

1. Bisognano, J., Wichmann, E.: On the duality condition for a Hermitian scalar field. J. Math. Phys. 16, 985 (1975)

2. Borchers, H.-J.: The CPT-Theorem in two-dimensional theories of local observables. Commun. Math. Phys. 143, 315 (1992)

3. Buchholz, D., Schulz-Mirbach, H.: Haag duality in conformal quantum field theory. Rev. Math. Phys. 2, 105 (1990)

4. Doplicher, S., Longo, R.: Standard and split inclusions of von-Neumann-algebras. Inv. Math. 75, 493 (1983)

5. Furlan, P., Sotkov, G.M., Todorov, I.T.: Two dimensional conformal quantum field theory. Riv. Nuovo Cimento 12, No. 6, 1 (1989)

6. Haag, R.: Local Quantum Physics. Berlin, Heidelberg, New York: Springer 1992

7. Jörß, M.: Lokale Netze auf eindimensionalen Lichtkegeln. Diploma thesis, FU Berlin (1991)

8, Schroer, B.: Recent developments of algebraic methods in quantum field theories. Int. J. Mod. Phys. B6, 2041 (1992)

9. Wiesbrock, H.-W.: Half-Sided Modular Inclusions of von-Neuman-algebras. Preprint FU Berlin (1992), to be published in Commun. Math. Phys.

10. Wiesbrock, H.-W.: Symmetries and Half-Sided Modular Inclusions of von-NeumannAlgberas. Preprint FU Berlin (1992), to be published in Lett. Math. Phys. 
